\title{
Inflammatory pseudotumor-like follicular dendritic cell tumor of the liver with expression of estrogen receptor suggests a pathogenic mechanism: a case report and review of the literature
}

\author{
Yanhui Liu $\cdot$ Li Li $\cdot$ Qinglong Hu $\cdot$ Roberto N. Miranda
}

Received: 20 February 2010 /Accepted: 6 September 2010 /Published online: 28 September 2010

(C) Springer-Verlag 2010

\begin{abstract}
The term inflammatory pseudotumor-like follicular dendritic cell tumor has been suggested for a follicular dendritic cell tumor of the liver associated with a dense inflammatory infiltrate and Epstein-Barr virus infection. This tumor is less aggressive than usual intraabdominal follicular dendritic cell sarcoma and shows a strong female predominance; however, the pathogenesis is uncertain. We report the case of a 59-year-old woman who presented with a 6-cm mass in the liver. Histological examination showed spindle cells with a storiform pattern and prominent inflammatory infiltrate mimicking inflammatory pseudotumor. Spindle cells expressed follicular dendritic cell markers CD21 and CD35, and were positive for EpsteinBarr virus-encoded RNA and LMP-1 protein. In addition, neoplastic cells expressed estrogen receptor (ER). Fifteen cases of inflammatory pseudotumor-like follicular dendritic
\end{abstract}

\footnotetext{
Y. Liu $\cdot$ L. Li

Department of Pathology and Laboratory Medicine,

Guangdong General Hospital and Guangdong Academy

of Medical Sciences,

106 2nd Zhongshan Road,

Guangzhou 510080, People's Republic of China

Q. $\mathrm{Hu}$

Department of Pathology, Heartland Regional Medical Center,

5325 Faraon Street,

Saint Joseph, MO 64506, USA

Q. Hu $(\bowtie)$

Physicians Reference Laboratory,

Overland Park, KS, USA

e-mail: qinglonghu@hotmail.com

R. N. Miranda

Department of Hematopathology, M.D. Anderson Cancer Center,

1515 Holcombe Blvd, Unit 072,

Houston, TX 77030, USA
}

cell tumor of the liver including the case we describe here have been reported in the literature. A review of the literature disclosed that normal follicular dendritic cells may express ER; however, extensive testing has not been previously reported in follicular dendritic cell sarcoma. The female predominance and the expression of ER in the case we report are suggestive of a hormonal role in the pathogenesis and therapy of inflammatory pseudotumorlike follicular dendritic cell tumor of the liver.

Keywords Inflammatory pseudotumor · Follicular dendritic cell tumor $\cdot$ Epstein-Barr virus $\cdot$ ER $\cdot$ Liver

\section{Introduction}

Inflammatory pseudotumor (IPT) encompasses a heterogeneous group of disorders, both nodal and extranodal, usually considered reparative, characterized by a prominent inflammatory infiltrate admixed with proliferating spindle cells, and historically considered as myofibroblasts [1]. Inflammatory pseudotumor involves diverse anatomic sites including lung, gastrointestinal tract, and lymph nodes [2]. A subset of lesions of inflammatory pseudotumor involving soft tissues of children and young adults have been reclassified as inflammatory myofibroblastic tumors because of clinical behavior of recurrence, the uniform cellularity of myofibroblasts including cellular atypia, and the presence of chromosome 2p23 translocations with overexpression of ALK-1 gene [3]. More recently, lesions with features of IPT mainly involving the liver and spleen were found to predominate in females, and composed of variable amounts of spindle cells with follicular dendritic cell or smooth muscle differentiation, however, clinically less aggressive than other intraabdominal follicular dendritic cell sarcoma. 
In addition, these tumors showed a strong association with Epstein-Barr virus (EBV) [4]. Because of these distinctive features, Cheuk et al. [5] suggested the term of inflammatory pseudotumor-like follicular dendritic cell tumor (IPT-FDCT) for these lesions. Clonality of episomal EBV genome, recurrence, and rare metastasis support the neoplastic nature of the hepatic lesions [6].

Although EBV appears involved, the pathogenesis of these lesions remains uncertain. Of 14 cases of IPT-FDCT of liver that have been reported in the English literature, there is a strong female predominance, suggesting that hormonal factors may play a pathogenic role. Herein, we report the case of a 59-year-old woman with IPT-FDCT of the liver who demonstrated expression of estrogen receptors (ER) in neoplastic spindle cells. Therefore, we searched the literature for the status of sex hormone receptors in published cases of IPT-FDCT of the liver and discuss the possible role of ER in the pathogenesis of this neoplasm.

\section{Report of a case}

An asymptomatic 59-year-old Chinese woman presented for a routine health check-up. Patient did not refer abdominal pain, fever, weight loss, or constitutional symptoms. Abdominal computed tomography scan revealed a mass lesion in the left hepatic lobe with features suspicious for hepatocellular carcinoma. Ultrasound examination also identified a $6.0 \times 5.2-\mathrm{cm}$ hepatic lesion, without other abdominal abnormalities. Chest X-ray result was normal. Laboratory testing showed normal liver function tests; serum $\alpha$-fetoprotein was $1.51 \mathrm{ng} / \mathrm{mL}$ (normal range, $<9.0 \mathrm{ng} / \mathrm{mL}$ ) and carcinoembryonic antigen was $2.9 \mathrm{ng} /$ $\mathrm{mL}$ (normal range, $<5.0 \mathrm{ng} / \mathrm{mL}$ ). Serologic testing results for hepatitis B surface antigen, and hepatitis A, C, D, and $\mathrm{E}$ antibodies were all negative. A partial hepatectomy was performed, and the mass lesion was entirely removed.

\section{Material and methods}

\section{Clinical history}

Clinical history was retrieved from the patient medical chart in the Office of Medical Record at Guangdong General Hospital, China. Clinical follow-up was performed through questionnaire form.
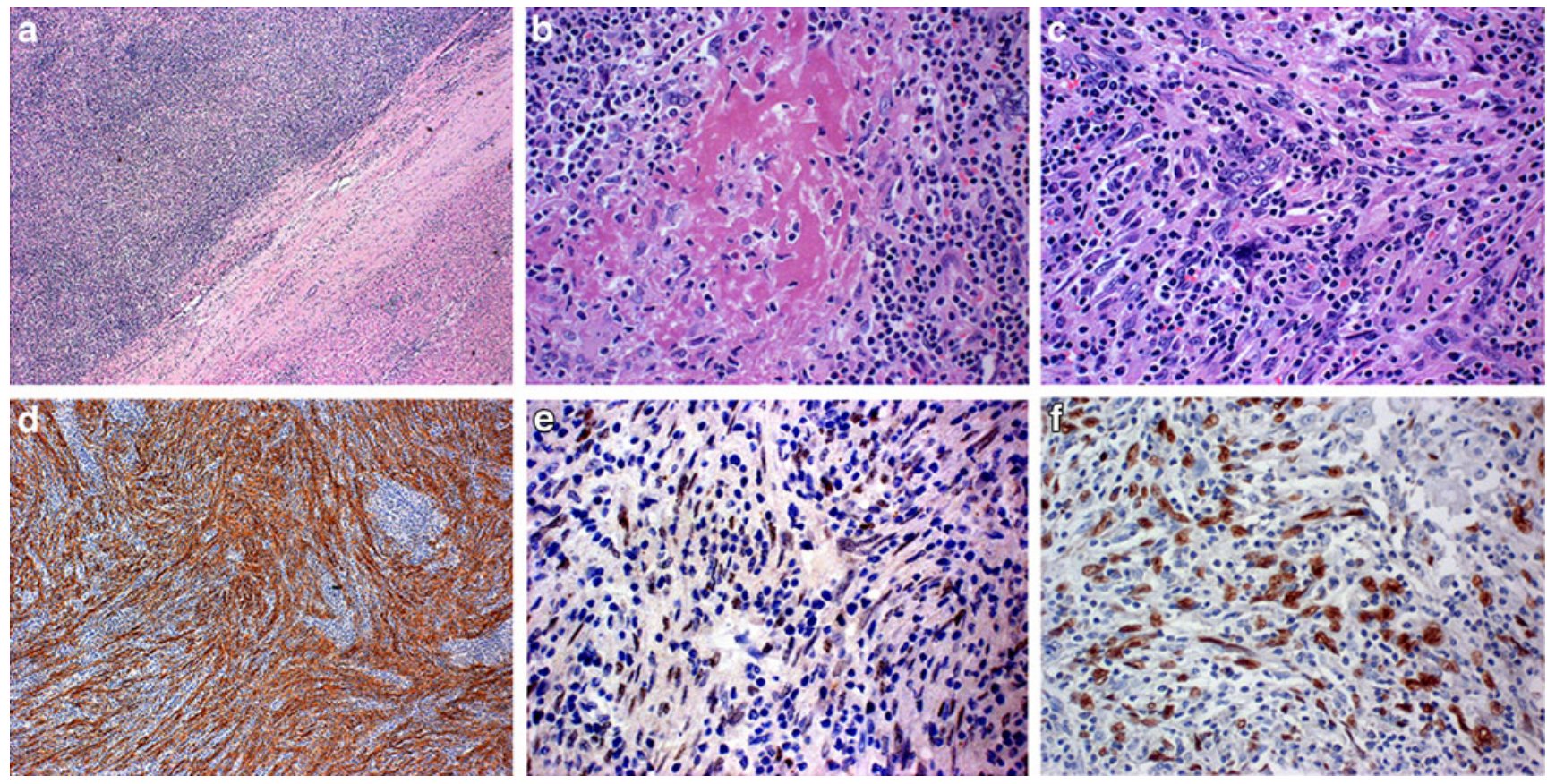

Fig. 1 a Inflammatory pseudotumor-like follicular dendritic cell tumor (IPT-FDCT) shows partial encapsulation with dense inflammatory infiltration; normal hepatic tissue present in the right lower field (H\&E stain, original magnification $\times 40$ ). b Foci of coagulative necrosis and hemorrhage are noted (H\&E stain, original magnification $\times 400)$. $\mathbf{c}$ The tumor shows spindle cells arranged in short fascicles mixed with infiltrate of small lymphocytes and plasma cells (H\&E stain, original

magnification $\times 400)$. d A storiform pattern formed by the spindle cells is highlighted by strong CD21 stain (original magnification $\times 100$ ). e Immunohistochemistry shows nuclei of the spindle cells positive for ER (original magnification $\times 400$ ). $\mathbf{f}$ In situ hybridization for EBER showing nuclear and cytoplasmic reactivity of the spindle cells (original magnification ×400) 
Table 1 Summary of immunophenotypic profile of the tumor cells

Histopathology

\section{Immunohistochemical studies}

The resected surgical specimen was sliced and then fixed in $10 \%$ buffered formalin. Representative sections were taken for routine histologic processing and paraffin embedding. The tissue blocks were cut into 4- $\mu \mathrm{m}$ sections and were stained with hematoxylin and eosin (H\&E). Immunohistochemical staining was performed on formalin-fixed and paraffin-embedded tissue sections with Avidin-biotin-peroxydase complex after antigen retrieval in a steamer. Antibodies from Dako, Denmark and other companies for the staining included ALK-1 (1:50), Bcl-2 (1:50), CD1a
(1:400), CD8 (1:100), CD20 (1:800), CD21 (1:50), CD23 (1:50), CD30 (1:100), CD34 (1:200), CD35 (1:20), CD45 (1:800), CD68 (1:200), CK (1:100), Desmin (1:50), EMA (1:200), ER (1:50), Granzyme B (1:200), Ki67 (1:100), MPO (1:8,000), p53 (1:500), PR (1:50), S-100 (1:2,000), SMA (1:100), Vimentin (1:200); CD4 (1:10), CD3 (Novacastra, Newcastle upon Tyne, UK,1:100), HMB45 (1:50), CD15 (Labvision, USA, 1:50), LMP-1 (Cell Marque, 1:1), and TIA-1 (Immunotech, 1:1,000). Appropriate positive and negative controls were evaluated simultaneously.

\section{In situ hybridization for $E B V$}

In situ hybridization for EBV-encoded small RNA (EBER) was performed on formalin-fixed, paraffin-embedded tissue sections using fluorescein isothiocyanate (FITC)-labeled peptic nucleic acid (PNA) probes against EBER (code Y5200; Dakopatts, Glostrup, Denmark). A positive signal was detected with a biotin-free, anti-FITC horseradish peroxidase-conjugated antibody. The reaction was performed with Dako PNA ISH detection Kit (Dako A/S). A known positive control was evaluated to ascertain the sensitivity of the assay.

\section{Ultrastructural analysis}

Multiple pieces of tissue sampled at about $1 \mathrm{~mm}$ from the tumor were fixed in 3\% glutaraldehyde and then post-fixed in $1 \%$ osmium tetroxide. The tissue embedded in epoxy resin was cut to $1 \mu \mathrm{m}$ of thickness and stained in toluidine blue solution. Appropriate blocks were chosen for ultrathin sectioning and the sections were stained with uranyl acetate and lead citrate. Electron microscopic examination was performed using a Philips CM 100 machine under $80 \mathrm{kV}$ accelerating voltage.
Fig. 2 An electron microscopic photograph shows ultrastructure of spindle cells with long villous cell processes and desmosomes (arrow). No complex junctions, cytoplasmic interdigitations, or Birbeck granules were identified

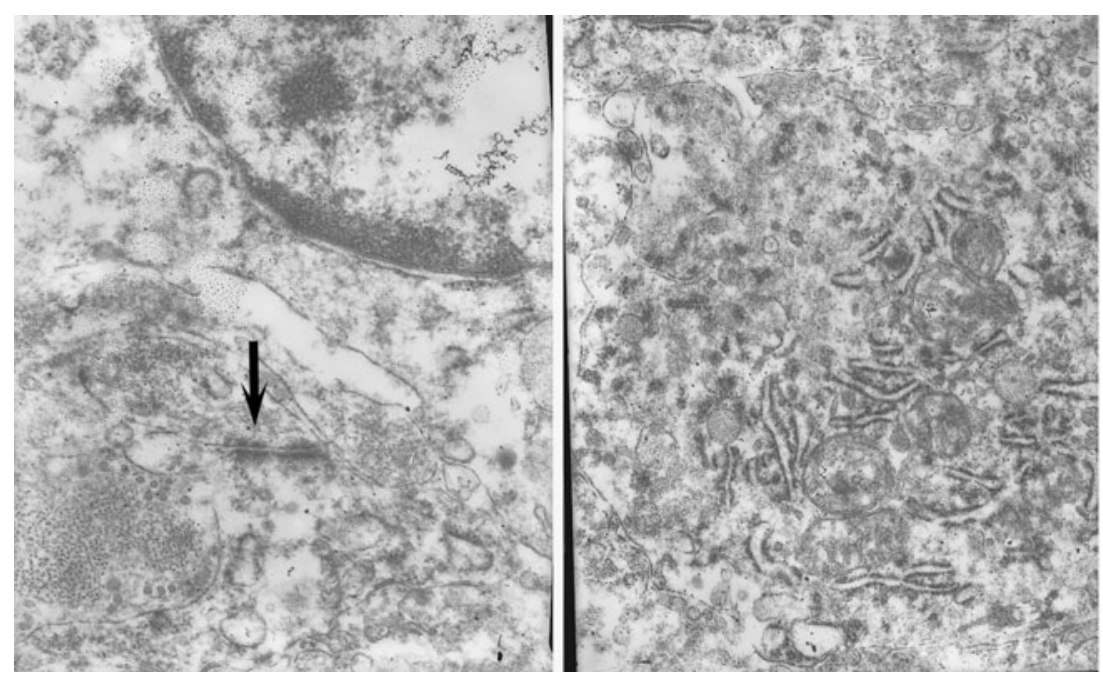




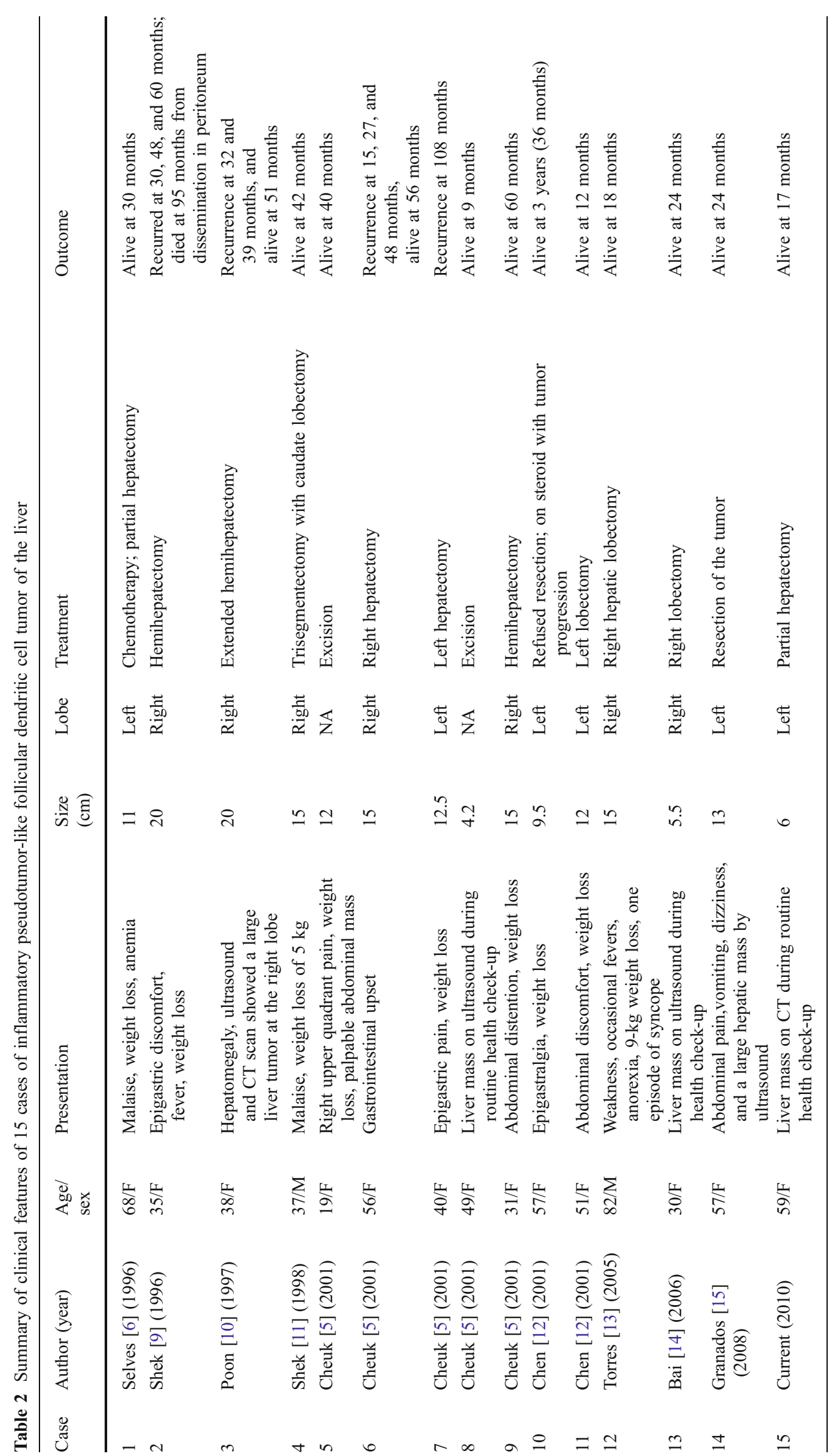




\section{Results}

Macroscopic findings

The resected specimen contained a well-circumscribed, unencapsulated mass lesion measuring $6.0 \times 4.5 \times 3.0 \mathrm{~cm}$. The cut surfaces were tan and fleshy with punctated dark red to yellow areas of hemorrhage and necrosis, respectively. No satellite nodules were identified.

\section{Histologic findings}

Routinely stained histologic sections showed a partially encapsulated lesion composed of spindle cells arranged in short fascicles with an associated inflammatory background composed of small lymphocytes and plasma cells. Some areas showed interlacing fascicles of spindle cells with pale pink cytoplasm and indistinct cell borders, as well as focal storiform pattern. The nuclei of the spindle cells were ovoid to fusiform, showing small or inconspicuous basophilic nucleoli (Fig. 1a, b and c) with no obvious atypia. Rare multinucleated giant cells were present. There were $0-1$ mitotic figures per ten high-power fields. Focal necrosis and hemorrhage were noted. The adjacent liver parenchyma was unremarkable, except for mild congestion.

\section{Immunophenotypic findings}

Immunohistochemical studies demonstrated that the spindle cells were diffusely and strongly positive for CD21 and CD35 (Fig. 1d). The spindle cells were positive for vimentin and ER (Fig. 1e), but were negative for progesterone receptors. The spindle cells, but not lymphocytes, were positive for EBV LMP-1, displaying cell membrane and cytoplasmic reactivity. Focal weak reactivity for p53 was noted. Spindle cells were negative for cytokeratin, EMA, CD15, CD30, CD68, HMB45, smooth muscle actin, desmin, ALK-1, S-100, BCL-2, CD34, CD45, $\mathrm{CD} 1 \mathrm{a}$, and myeloperoxidase. The proliferation fraction as determined by Ki-67 was about $1 \%$. The background small lymphocytes were predominantly CD8-positive T cells. The majority of the $\mathrm{T}$ cells expressed intracellular $\mathrm{T}$ cell antigen 1 (TIA-1) and granzyme B. Scattered small lymphocytes were positive for CD20 (see Table 1 for summary of the results).

In situ hybridization for EBER showed an intense nuclear and cytoplasmic stain in many of the spindle cells (Fig. 1f). The background small lymphocytes and plasma cells were negative.

Electron microscopic findings

Ultrastructural examination showed spindle cells with long villous cell processes and desmosomes. No complex junctions, cytoplasmic interdigitations, or Birbeck granules were identified (Fig. 2).

\section{Discussion}

IPT-FDCT of the liver is a recently described rare tumor and considered as a variant of follicular dendritic cell sarcoma, with predominance in females, however, clinically less aggressive than usual intraabdominal follicular dendritic cell sarcoma. It is characterized by a mixture of chronic inflammatory cells and variable amounts of spindle cells with follicular dendritic cell differentiation, usually expressing EBV. IPT-FDCT is distinct from the more common IPT which is a reparative process that involves diverse anatomic sites, including lung and gastrointestinal tract [2], and when involving liver, usually is associated with bile duct obstruction and can regress with antibiotic therapy $[7,8]$.

Fifteen cases of hepatic IPT-FDCT, including the case we report, have been identified since the first case of hepatic IPT-FDCT was reported by Selves et al. [6] (Tables 2 and 3) [5, 6, 9-15]. Similar features of IPT-FDCT of the liver have been found in the spleen. Patients with IPT-FDCT of the liver show an age range of 19-82 years with a median age of 49 years. Female cases predominate (female to male ratio, 6.5:1). CT scan or ultrasound examination usually shows a solitary, well-circumscribed mass in the liver,

Table 3 Immunohistochemical profile of 15 cases of inflammatory pseudotumor-like follicular dendritic cell tumor of the liver

\begin{tabular}{llr}
\hline Markers & Number of cases (+/total) & Positive (\%) \\
\hline Actin & $1 / 2$ & 50 \\
ALK & $0 / 7$ & 0 \\
CD1a & $0 / 1$ & 0 \\
CD21 & $10 / 10$ & 100 \\
CD21/CD35 & $5 / 5$ & 100 \\
CD23 & $7 / 12$ & 58 \\
CD35 & $7 / 9$ & 78 \\
CD68 & $1 / 4$ & 25 \\
Clusterin & $1 / 1$ & 100 \\
CNA.42 & $8 / 8$ & 100 \\
Desmin & $0 / 3$ & 0 \\
EBER & $13 / 14$ & 93 \\
Ki-M4P & $3 / 3$ & 100 \\
LMP-1 & $7 / 8$ & 86 \\
R4/23 & $2 / 2$ & 100 \\
S-100 & $0 / 6$ & 0 \\
SMA & $7 / 12$ & 58 \\
Vimentin & $5 / 5$ & 100 \\
\hline
\end{tabular}


measuring from 4 to $20 \mathrm{~cm}$ in greatest dimension. Surgical resection was performed in 14/15 patients; one received chemotherapy for Hodgkin lymphoma because of misdiagnosis before surgical resection; one refused resection and her tumor showed slow progression despite steroid therapy. Eleven of $15(73 \%)$ patients are alive with no recurrence after 3-60 months follow-up after surgical removal. Local recurrence was observed in the remaining four $(27 \%)$ patients, 15 to 108 months after initial resection. Only one patient was reported dead of intra-hepatic and peritoneal dissemination 95 months after surgery.

A review of the immunohistochemical reactivity of 15 cases [5, 6, 9-15] revealed that $10 / 10(100 \%)$ cases were positive for $\mathrm{CD} 21,7 / 9(78 \%)$ positive for $\mathrm{CD} 35$, and five cases were either strongly positive or partial weak positive for a CD21/CD35 cocktail. Expression for other follicular dendritic cell markers was also observed, including 8/ $8(100 \%)$ for CNA.42, 3/3 (100\%) for Ki-M4P, and $2 / 2$ (100\%) for R4/23. Variable expression of muscle-associated antigens was noted: $1 / 2(50 \%)$ for actin, $6 / 9(67 \%)$ for HHF35, and 1/3 (33\%) for smooth muscle actin. No desmin expression was identified in three tested cases. The neoplastic cells were negative for ALK-1, S100, EMA, and cytokeratin.

The pathogenic mechanism of EBV in IPT-FDCT of the liver is unclear as IPT-FDCT of spleen and IPT. IPT comprises a group of heterogeneous diseases; a portion of the disease is associated with abnormal function of ALK1 and some are associated with EBV infection. The progenitor cells infected by EBV appear different in IPT from those in IPT-FDCT. Our report supports that IPT-FDCT belongs to EBV-associated neoplasms. Most hepatic IPTFDCT was associated with EBV, which specifically infected spindle-appearing dendritic cells. EBER and/or LMP-1 were reported in 13/14 (93\%) of cases. The most sensitive mean of detecting EBV infection in tissues is through EBER testing, while LMP-1 immunohistochemistry is less sensitive [4]. LMP-1 gene is considered an EBV oncogene and is capable to transform rodent fibroblasts in vitro. EBV genome with point mutations or 30-bp deletion in exon 3 of LMP-1 gene is prevalent in Asia and was identified in one of two hepatic IPT-FDCT reported by Chen et al. [12]. No EBV was identified in 24 cases of extranodal FDCS reviewed by Shia [16]. EBV was identified by LMP-1 or EBER only in the neoplastic spindle cells, but not in the lymphocytes or plasma cells, suggesting a pathogenic association of EBV. Clonal EBV genome was identified in hepatic IPT-FDCT [6, 12], which suggests that EBV infection was an early event in the transformation of the tumor precursor cells. However, multiple genetic mutations are required for malignant transformation, which are largely unknown for IPT-FDCT.

Since there is marked female predominance among cases of IPT-FDCT of the liver, it is tempting to suggest that female hormones or their receptors may contribute to growth of follicular dendritic cell tumors infected by EBV. A synergistic role of EBV and ER is speculated in the pathogenesis of IPT-FDCT; however, no evidence has been identified about this and studies about the interaction of EBV and ER have not been reported in literature. Sapino et al. [17] reported ER protein overexpression and increased mRNA of ER in normal follicular dendritic cells, which paradoxically became more prominent after tamoxifen therapy in cases with breast cancer. They also reported negativity for ER of two cases of follicular dendritic cell sarcoma. Our review of the literature did not reveal other reports of ER status in follicular dendritic cell sarcoma or in IPT-FDCT. The significance of ER expression in this case of IPT-FDCT of the liver raises the possibility that ER contributed to tumor growth. In comparison, it is acknowledged that desmoids tumor, also known as extraabdominal fibromatosis, also has a strong female predominance and ER overexpression in approximately $90 \%$ of cases; tamoxifen has been reported effective in adjuvant therapy of desmoids tumor, suggesting that the tumor is hormonedependent [18]. If the association between IPT-FDCT and ER overexpression is confirmed, ER could be evaluated as a potential therapeutic target.

In summary, IPT-FDCT of the liver is a neoplasm of follicular dendritic cells admixed with abundant inflammatory cells and shows a strong association with EBV infection. It is less aggressive than the usual intraabdominal follicular dendritic cell sarcoma, but shows recurrences, thus surgical resection is the treatment of choice. The pathogenesis is uncertain and is puzzling in its strong predominance in females, thus, we tested one of these tumors for ER and demonstrated its expression in the tumor cells, similar to what occurs in normal follicular dendritic cells, but apparently not in follicular dendritic sarcoma as reported previously. This finding warrants a systematic evaluation for ER in follicular dendritic cell sarcoma and IPT/FDCT because of the potential for a specific therapeutic target.

Acknowledgement We thank Dr. Hengguo Zhuang and Dr. Minghui Zhang for diagnostic consultation and are grateful to Xinlan Luo for expert technical assistance.

Conflict of interest The authors declare that they have no conflict of interest.

\section{References}

1. Bahadori M, Liebow AA (1973) Plasma cell granulomas of the lung. Cancer 31:191-208

2. Moran CA, Suster S, Abbondanzo SL (1997) Inflammatory pseudotumor of lymph nodes: a study of 25 cases with emphasis on morphological heterogeneity. Hum Pathol 28:332-338 
3. Coffin CM, Watterson J, Priest JR, Dehner LP (1995) Extrapulmonary inflammatory myofibroblastic tumor (inflammatory pseudotumor). A clinicopathologic and immunohistochemical study of 84 cases. Am J Surg Pathol 19:859-872

4. Arber DA, Weiss LM, Chang KL (1998) Detection of EpsteinBarr Virus in inflammatory pseudotumor. Semin Diagn Pathol $15: 155-160$

5. Cheuk W, Chan JK, Shek TW, Chang JH, Tsou MH, Yuen NW, Ng WF, Chan AC, Prat J (2001) Inflammatory pseudotumor-like follicular dendritic cell tumor: a distinctive low-grade malignant intra-abdominal neoplasm with consistent Epstein-Barr virus association. Am J Surg Pathol 25:721-731

6. Selves J, Meggetto F, Brousset P, Voigt JJ, Pradere B, Grasset D, Icart J, Mariame B, Knecht H, Delsol G (1996) Inflammatory pseudotumor of the liver. Evidence for follicular dendritic reticulum cell proliferation associated with clonal Epstein-Barr virus. Am J Surg Pathol 20:747-753

7. Goldsmith PJ, Loganathan A, Jacob M, Ahmad N, Toogood GJ, Lodge JP, Prasad KR (2009) Inflammatory pseudotumours of the liver: a spectrum of presentation and management options. Eur J Surg Oncol 35:1295-1298

8. Park KS, Jang BK, Chung WJ, Cho KB, Hwang JS, Kang YN, Kang KJ, Kim MJ, Kwon JH (2006) Inflammatory pseudotumor of livera clinical review of 15 cases. Korean J Hepatol 12:429-438

9. Shek TW, Ho FC, Ng IO, Chan AC, Ma L, Srivastava G (1996) Follicular dendritic cell tumor of the liver. Evidence for an Epstein-Barr virus-related clonal proliferation of follicular dendritic cells. Am J Surg Pathol 20:313-324

10. Poon RT, Shek TW, Lo CM (1997) Repeated hepatectomies for recurrent follicular dendritic cell tumour of liver: a case report. Eur J Surg Oncol 23:572-574
11. Shek TW, Liu CL, Peh WC, Fan ST, Ng IO (1998) Intraabdominal follicular dendritic cell tumour: a rare tumour in need of recognition. Histopathology 33:465-470

12. Chen TC, Kuo TT, Ng KF (2001) Follicular dendritic cell tumor of the liver: a clinicopathologic and Epstein-Barr virus study of two cases. Mod Pathol 14:354-360

13. Torres U, Hawkins WG, Antonescu CR, DeMatteo RP (2005) Hepatic follicular dendritic cell sarcoma without Epstein-Barr virus expression. Arch Pathol Lab Med 129:1480-1483

14. Bai LY, Kwang WK, Chiang IP, Chen PM (2006) Follicular dendritic cell tumor of the liver associated with Epstein-Barr virus. Jpn J Clin Oncol 36:249-253

15. Granados R, Aramburu JA, Rodriguez JM, Nieto MA (2008) Cytopathology of a primary follicular dendritic cell sarcoma of the liver of the inflammatory pseudotumor-like type. Diagn Cytopathol 36:42-46

16. Shia J, Chen W, Tang LH, Carlson DL, Qin J, Guillem JG, Nobrega J, Wong WD, Klimstra DS (2006) Extranodal follicular dendritic cell sarcoma: clinical, pathologic, and histogenetic characteristics of an underrecognized disease entity. Virchows Arch 449:148-158

17. Sapino A, Cassoni P, Ferrero E, Bongiovanni M, Righi L, Fortunati N, Crafa P, Chiarle R, Bussolati G (2003) Estrogen receptor alpha is a novel marker expressed by follicular dendritic cells in lymph nodes and tumor-associated lymphoid infiltrates. Am J Pathol 163:1313-1320

18. Santos GA, Cunha IW, Rocha RM, Mello CA, Guimraes GC, Fregnani JH, Lopes A (2010) Evaluation of estrogen receptor alpha, estrogen receptor beta, progesterone receptor and cKit expression in desmoids tumors and their role in determining treatment options. Biosci Trends 4:25-30 